\title{
EDITORIAL
}

\section{Stress and myocardial infarction}

\section{J S Chi, R A Kloner}

Could there be a relation between cardiovascular events and environmental stress in the form of sporting events, making winning or losing a life or death issue?

M yocardial infarction (MI) is a serious health problem which causes substantial morbidity and mortality. The seriousness of coronary artery disease is heightened by the fact that approximately a third of patients present with sudden death as their first manifestation, ${ }^{1}$ and efforts to prevent infarctions before they occur have fuelled an area of research which investigates inciting events, or "triggers", of MI, arrhythmias, and sudden death. The idea that an MI can be incited by an identifiable event is an old one, but research into cardiovascular triggers has only recently begun to elucidate specific culprits. Triggering research continues to evolve in concert with our understanding of the pathophysiological mechanisms involved in MI, with the eventual hope of developing effective preventive strategies.

The circadian rhythm in the incidence of cardiac events was one of the first triggering patterns to be discovered; peak frequencies of MI and sudden cardiac death were observed in the morning hours after waking. ${ }^{2}$ Routine activities of daily life such as physical exertion and smoking, ${ }^{4}$ as well as a variety of negative emotions such as anger, tension, and sadness, ${ }^{5}$ were found to induce myocardial ischaemia. Sexual activity doubles the risk of MI, but because of a low absolute baseline risk and infrequent risk exposure, the proportion of MIs actually triggered by sexual activity is low at about $1 \%{ }^{6}$ Investigations into triggering have also extended into disasters, both man-made and natural. Studies have found an increased frequency of sudden cardiac death and MI in relation to catastrophes such as an earthquake ${ }^{7}$ or war, ${ }^{8}$ suggesting that psychological and emotional stress brought on by such disasters can trigger cardiac events.

\section{MYOCARDIAL INFARCTION AND SPORTING EVENTS}

Recently, attention has shifted toward a possible relation between cardiovascular events and environmental stress in the form of sporting events, with the hypothesis that the emotional intensity of important sporting events and other behaviours associated with spectating such as smoking, binge drinking, and overeating could trigger MIs. Football (soccer) has often been the focus of such studies since the intensity of football games is arguably unmatched by few other sporting events. Not surprisingly, all of the studies have been from Europe. One of the first studies was from the Netherlands, ${ }^{9}$ in which mortality from coronary heart disease and stroke was found to be increased in men on the day of an important football match between the Netherlands and France in the 1996 European football championship tournament. There was no similar increase found in women, nor on any of the other days in which the Dutch played a football game in that tournament. The increase occurred on the day the Dutch played their one game that ended in a penalty shoot-out, a do-or-die situation in which the winner of the game is determined in dramatic fashion. The study investigators proposed that the heightened intensity of the game and resultant outcome (the Dutch lost) may have been responsible for the 14 excess deaths caused by coronary heart disease and stroke in the Netherlands that day. A French group conducted a similar study in France $^{10}$ for the same day and did not find any increases in all cause mortality or mortality from MI or stroke, suggesting that the outcome of the game could have been a possible factor in triggering. Recently, Brunekreef and Hoek ${ }^{11}$ found no excess mortality from MI or stroke around five important football games played by the Dutch between 1988 and 1994. However, mortality was increased non-significantly in the one match that was decided by a penalty shoot-out.

A subsequent study from England ${ }^{12}$ showed that admissions from acute MI were increased by $25 \%$ on the day of and two days after England lost to Argentina in the 1998 World Cup in yet another game that ended in a penalty shoot-out. The game, which was the most watched television programme in England that year, eliminated England from the tournament. The increased number of admissions was found across both sexes, and there were no increases in admission as a result of any other diagnoses such as stroke, self-harm, or injuries from traffic accidents, making it less likely that the increase in MIs was caused by behaviours associated with watching the game such as excessive drinking, smoking, or eating. On a lighter note, the apparent isolation of excess adverse cardiovascular events to games which end in a penalty shoot-out compelled the authors to suggest: "perhaps . . the penalty shoot-out should be abandoned on public health grounds", a suggestion which in all likelihood would not be well received by football fans.

\section{INCREASED SYMPATHETIC ACTIVITY}

Potential mechanisms by which watching sporting events could contribute to cardiac events are increases in sympathetic nervous system stimulation and circulating catecholamines induced by the emotional involvement in the game. Increases in heart rate and blood pressure have been recorded in viewers watching sporting events, ${ }^{13}$
Wilshire Blvd

CA 90017, USA

rkloner@goodsam.org 
supporting this notion. Increased sympathetic stimulation and catecholamines increase oxygen demand by increasing heart rate, blood pressure, and cardiac contractility; stimulation of $\alpha$ sympathetic receptors can increase coronary vascular tone, reducing relative oxygen supply. In addition, increased ventricular inotropy and changes in coronary tone may alter the shear stress of blood against a vulnerable atherosclerotic plaque, contributing to plaque fracture. Finally, increased concentrations of catecholamines can trigger arrhythmias and increase platelet aggregation.

The study by Berthier and Bouley in this issue of Heart ${ }^{14}$ adds to this small body of growing evidence linking sporting events and cardiovascular events. Their study, again centred around an important football match, this time in France, found a significant decrease in mortality from MI in men on the day France won the 1998 World Cup of football, with a similar but non-significant decrease in women. The resultant euphoria after the victory was heightened by the fact that the game took place in France. The authors hypothesise that the observed phenomenon may have been caused by a decreased level of activities during the day of the game and the "euphoria" of victory. Interestingly, there was also a decrease in mortality from MI in men two days after the game, on 14 July, a national holiday in France. The authors suggest that a day off of work may have been a contributing factor; indeed, the incidence of MI has been shown to be highest on Mondays, possibly from the stress of having to return to work.

The decrease in mortality from MI in this study was only found in men, whereas previous triggering studies have typically found that men and women are equally vulnerable to triggers. The equivocal results in the women might be explained by sex differences with respect to watching sports-for example, fewer women may have watched the game, or women may not have been as emotionally involved in its outcome. The authors also observed that while mortality from MI in men decreased, all cause mortality remained relatively stable. It would be interesting to know what, if any, specific causes were responsible for compensating the decrease in MI mortality. Also, the magnitude of the decrease in MI mortality was the same on the day of the game and a national holiday two days later. We cannot rule out the possibility that the decreased mortality seen on the game day was caused by simply not going to work. Obviously, speculation on observed trends can continue at great length, and the difficulty in teasing out specific inciting factors is an unavoidable drawback of this type of study.

\section{DECREASED MORTALITY}

However, Berthier and Boulay's study is notable because it shows a decrease in mortality around the time of a positive life event, which, as they note, is the first time a positive effect has been seen from a sporting event. Indeed, previous triggering studies have primarily focused on negative emotions as a possible trigger for cardiac events, and there is much less evidence on the possible effects of positive emotions. The idea that positive feelings could modify the risk of cardiovascular events is an intriguing one, and future studies that clarify any possible link would certainly be of great interest.

Triggering studies are valuable for informing patients of their risk of ischaemic events during particular activities and counselling them on reducing their risk. Previous studies have shown that the regular use of certain medications such as aspirin was associated with a reduced risk of ischaemia during an ischemia inducing episode..$^{15}$ At this point, it is somewhat preliminary to base recommendations for lifestyle or behaviour changes on the results of the sports studies; certainly, the most prudent approach is still the optimisation of each patient's lifestyle, particularly diet and exercise, as well as any necessary medication regimen. Research in the field of triggering related to sporting events is intriguing and should continue, since fans may or may not be losing their lives after football games, but football games are not losing any fans.

\section{Authors' affiliations}

J S Chi, R A Kloner, The Heart Institute, Good Samaritan Hospital, Division of Cardiovascular Medicine, Keck School of Medicine, University of Southern California, Los Angeles, California, USA

\section{REFERENCES}

1 Friesinger GC. The natural history of atherosclerotic coronary heart disease. In: Schlant RC, Alexander RW, ed. Hurst's the heart, 8th ed. New York: McGraw-Hill, 1994:1199.

2 Muller JE, Ludmer PL, Willich SN, et al. Circadian variation in the frequency of sudden cardiac death. Circulation 1987;75:131-8.

3 Muller JE, Stone PH, Turi ZG, et al. Circadian variation in the frequency of onset of acute myocardial infarction. N Engl J Med 1985;313:1315-22.

4 Gabbay FH, Krantz DS, Kop WJ, et al. Triggers of myocardial ischemia during daily life in patients with coronary artery disease: physical and mental activities, anger and smoking. J Am Coll Cardiol 1996;27:585-92

5 Gullette ECD, Blumenthal JA, Babyak M, et al. Effects of mental stress on myocardial ischemia during daily life. JAMA 1997;277:1521-6.

6 Muller JE, Mittleman MA, Maclure M, et al. Triggering myocardial infarction by sexual activity. JAMA 1996:275:1405-9.

7 Leor J, Poole WK, Kloner RA. Sudden cardiac death triggered by an earthquake. N Engl J Med 1996;334:413-9.

8 Meisel SR, Kutz I, Dayan K, et al. Effect of Iraqi missile war on incidence of acute myocardial infarction and sudden death in Israeli civilians. Lancet 1991;338:660-1.

9 Witte DR, Bots ML, Hoes AW, et al. Cardiovascular mortality in Dutch men during 1996 European football championship: longitudinal population study. BM 2000;321:1552-4.

10 Toubiana L, Hanslik T, Letrilliart L. French cardiovascular mortality did not increase during 1996 European football championship. BM 2001;322:1306.

11 Brunekreef B, Hoek G. No association between major football games and cardiovascular mortality. Epidemiology 2002;13:491-2.

12 Carroll D, Ebrahim S, Tilling K, et al. Admissions for myocardial infarction and World Cup football: database survey. BM 2002;325: 1439-42.

13 Harrison LK, Carroll D, Burns VE, et al. Cardiovascular and secretory immunoglobulin A reactions to humorous, exciting, and didactic film presentations. Biol Psychol 2000;52:113-26.

14 Berthier F, Boulay F. Lower myocardial infarction mortality in French men the day France won the 1998 World Cup of football. Heart 2003:89:555-6.

15 Mittleman MA, Maclure M, Sherwood J, et al for the Determinants of Myocardial Infarction Onset Study Investigators. Triggering of acute myocardial infarction onset by episodes of anger. Circulation 1995;92:1720-5. 